\title{
Development of an application for mobile devices with augmented reality for the teaching of fracture characteristics
}

\author{
Jose Alonso Oviedo Monroy ${ }^{a}$, Andrés Mauricio Arciniegas ${ }^{b}$ \\ ${ }^{a}$ Servicio Nacional de Aprendizaje SENA, Ibagué - Tolima, Colombia, jaoviedom@sena.edu.co \\ bervicio Nacional de Aprendizaje SENA, Ibagué - Tolima, Colombia, andarciniegas@sena.edu.co
}

\begin{abstract}
Introduction: this article is a product of the innovation project "Integration of augmented reality in the teaching-learning process of bone injuries", developed during 2018 in the Trade and Services Center of the National Training Service SENA in Tolima. Problem: The Trade and Services Center, and the technological network of health services does not have an easily accessible tool that allows you to permanently develop a knowledge check against possible cases of polytraumatism and the incidence of fractures in front of the epidemiological profile making an identification of the characteristics of the same. Objective: Integrate augmented reality tools in the teaching-learning process of bone injuries. Methodology: Descriptivepropositive of qualitative nature, descriptive when skeletal lesions were characterized and propositive as soon as an application for mobile devices was generated aimed at improving the teaching-learning process of fractures. Results: a mobile application based on Augmented Reality was developed, which supports the teaching-learning process of bone injuries. Conclusions: three-dimensional models of bones and bone lesions have been recreated, allowing trainees to assimilate their characteristics and primary management effectively, thus reinforcing their learning through interactive contents. Limitations: the project only covers the learning of bone injuries.
\end{abstract}

Keywords: Augmented Reality, teaching, learning, mobile application, fracture. 
Development of an application for mobile devices with augmented reality for the teaching of fracture characteristics

\section{Introduction}

As well as that society and technological developments evolve, so must education. Generational changes are nothing new and in education there will always be changes and challenges. Some educators have the belief that students of today's medical sciences are different from those of previous generations only because of their generational base (Twenge, 2009). Most of the apprentices of the careers of the health area, were born between 1977 and 1995, that is they belong to the "Generation Y", also known as "Millennials". Apparently, this generation has as its main characteristics its assertiveness, self-esteem, narcissistic features, high expectations and some features of stress, anxiety and poor mental health, as well as less dependence on themselves. On the other hand, their teachers, in general, belong to Generation X, so they are born from 1965 to 1976 or Baby Boomers (born between 1946 and 1964).

Therefore, knowing that each generation sees the world in a specific way and this is influenced by technology, world events and social norms, and that Millennials have grown up in an environment of unlimited information and seeking technology-based learning, it can be considered, then, that teaching supported only on PowerPoint slides is obsolete; therefore, contemporary teaching strategies must include e-learning, inverted classes, simulation, peer teaching, social networks, among others. (Hopkins, y otros, 2018).

Now, in the teaching of medicine, and particularly of bone lesions, traditional methodologies predominate. The master class continues to be the dominant pedagogical practice in university centers, therefore, the redesign of its objectives and contents becomes necessary (Carretero González, 2010). In this order of ideas, the discussion of practical cases, problem solving, collaborative learning, interactive and other active learning techniques should be potentiated. The Trade and Services Center of the National Training Service SENA Regional Tolima and the technological network of health services, does not have a tool that allows to develop the verification of knowledge regarding cases of polytraumatisms and the incidence of fractures against the epidemiological profile, making an identification of the characteristics of these. In particular, the predominant methodology currently (for the technological program of Diagnostic Imaging) is teaching through the study of cases with a few radiographs and tomographies..

In order to delve into the subject that concerns us, it is necessary to understand some things about the human body. One of the most important functions of the human being is locomotion. For this purpose, it requires a system composed of bones, ligaments and joints, which is called musculoskeletal. This system also fulfills other tasks such as protection and stability. However, as in any system, problems may arise that affect its normal functioning. One of these problems is bone injuries, which are "damaged caused to the structure or function of the body by an outside agent or force, which may be physical or chemical. 
Injury occurs when an acting force exceeds the elastic, plastic, viscoelastic, or endurance limits of a tissue" (Bulstrode et al., 2005).

Continuing with the definitions that help solve the problem, it is found that one that indicates that augmented reality (AR) is the direct or indirect view in real time of the physical environment of the real world that has been improved (increased) by adding information virtual computer generated (Furht, 2011). Augmented reality is an environment that merges real objects with digital 3D objects, then, by joining virtual and real worlds, augmented reality creates an enhanced and enhanced reality (Kippe \& Rampolla, 2013). On the other hand, Azuma (1997) defines it as a system that fulfills three basic characteristics: a combination of real and virtual worlds, real-time interaction and an accurate $3 \mathrm{D}$ record of virtual and real objects.

According to Klopfer y Squire (2008) the AR could be defined, in general terms, as a situation in which a real-world context dynamically overlaps with a coherent location or virtual information sensitive to the context.

Defining the AR in a broad sense would be more productive because such a definition suggests that it could be created and implemented by various technologies, such as desktops, portable devices, head-mounted displays, glasses, among others. That is to say, the notion of AR is not limited to any type of technology and could be reconsidered from a broad perspective at present. This is how augmented reality exploits the possibilities of the real world by providing additional and contextual information that increases the experience of students' reality (Klopfer \& Squire, 2008) (Wu, Wen-Yu Lee, Chang, \& Liang, 2013).

Ultimately, it can be concluded that augmented reality is the superposition of data through $3 \mathrm{D}$ spaces to produce a new experience of the world, amplifying access to information and generating new opportunities for learning.

To help to enhance interactive active didactics and for medical science education to migrate to the new learning paradigm (Kurup, 2010), arises the project to teach bone injuries through augmented reality, which aims to create an application for mobile devices that allow the student to visualize information of the different models of traumas, along with their description, so this one interacts using the glasses of augmented reality; perform recognition of knowledge through the generation of random questions, keep track of recognition of knowledge and send the latter through email to your teacher. 
Development of an application for mobile devices with augmented reality for the teaching of fracture characteristics

\section{Proposed solution}

The aim of the developed system is to improve the teaching - learning process of bone injuries in the technological training programs of Diagnostic Imaging, Nursing Assistant and Sports Training offered by the National Training Service SENA. For this reason, it is intended to be used by young people between 16 and 25 years, on average, who are interested in learning about the basic concepts of bone injuries.

This application works, mainly, on mobile devices with Android operating system whose version must be equal to or greater than 4.4 and iOS systems since version 9.0, since it uses Augmented Reality to visualize the different trauma models.

\subsection{Design}

As stated above, the objective of this project is to create an application to run on mobile devices that work with Android and iOS operating systems, which, through augmented reality, allows an apprentice to visualize the bones of the skeletal system and the fractures or dislocations, that under the criterion of orthopedic doctors and experts in the area, can be presented on these.

Fig. 1 shows the functionality of the application summarized in a diagram of use cases.

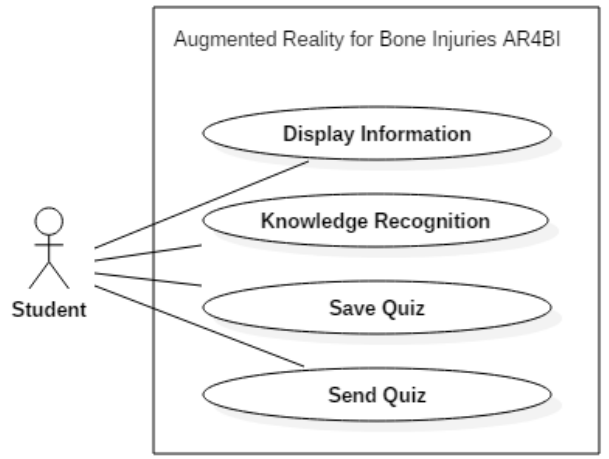

Fig. 1 Diagram of cases of use of the application

Thus, the functionality of the software is summarized in the following aspects:

- Visualization of different models of bone lesions using augmented reality. It allows the trainee to select a trauma model, deploy it along with its description and interact freely with the selected model making use of augmented reality goggles.

- To evaluate the knowledge acquired by the apprentice in the recognition of bone lesions. The application randomly selects a trauma model with a set of response options so that the apprentice, based on the learning obtained, takes one of the options deployed. After this, the application validates the answer and displays the result obtained. 
- Save recognition results. The application allows the trainee to be stored locally, by filing a recognition of previously developed knowledge in a relational database.

- Sending stored tracks through email. The trainee selects the saved traces that he wants to send, providing the recipient's data.

\section{Implementation}

\subsection{Architecture}

The application was developed using a closed multilayer architecture divided into 3 levels: persistence, business logic and presentation; in order to reduce the dependence between the different layers and improve their coupling, in such a way that each of them uses the characteristics only of the immediately lower one, in order to facilitate the maintenance and extension of the product.

\subsubsection{Persistence layer}

In the persistence layer were defined the procedures to access a local relational database, which is constituted in the data source, to store knowledge recognitions made by the learner and retrieve them for later reading and organized in a format for sending by email to the teacher.

Fig. 2 shows the relationship entity diagram that represents the storage structure of knowledge recognitions.

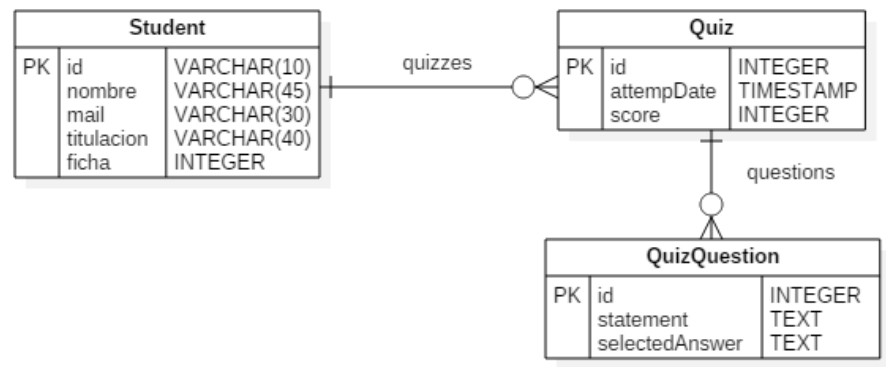

Fig. 2 Entity relationship diagram of the application

\subsubsection{Logical business layer}

In the business logic layer, the $\mathrm{C}$ \# programming language was used under the Microsoft Visual Comunity development environment, where the application's functionality was implemented. Therefore, in this layer was defined the procedures to control the interaction of the apprentice in the display of information on the screen when selecting a type of bone injury, in the conformation of evaluations for the recognition of knowledge, selection of 
Development of an application for mobile devices with augmented reality for the teaching of fracture characteristics

answers and validation of the these; in the control of the storage of recognition results and its sending by email through communication with the persistence layer.

Fig. 3 shows the class diagram of the application which represents the structural classification of the functionality of the system, that is, its organization based on functional units called classes and the existing associations between them at the level of this layer.

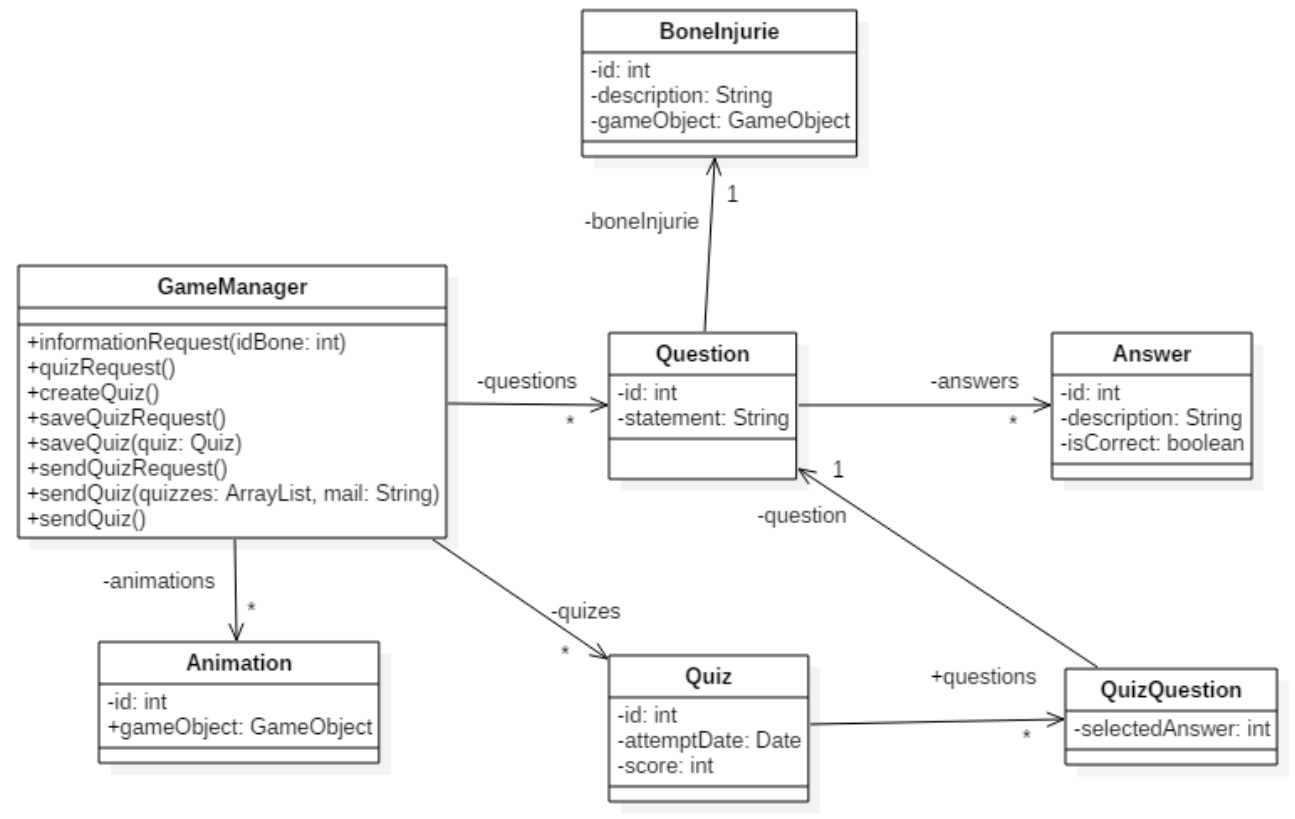

Fig. 3 Class diagram of the application

Fig. 4 displays the sequence diagram for knowledge recognition functionality, that is, the dynamic behavior of the system for the evaluation of the knowledge acquired by the apprentice in the recognition of bone injuries.

Now, in Fig. 5 presents the sequence diagram for the functionality of sending knowledge recognition previously stored by the apprentice, as shown in the previous figure. 


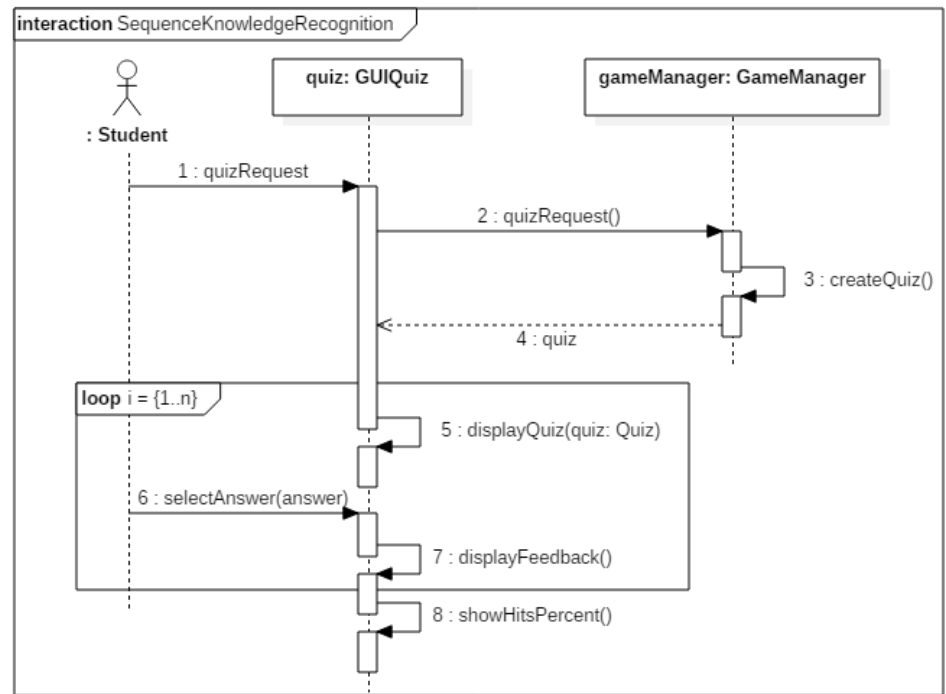

Fig. 4. Sequence diagram for knowledge recognition

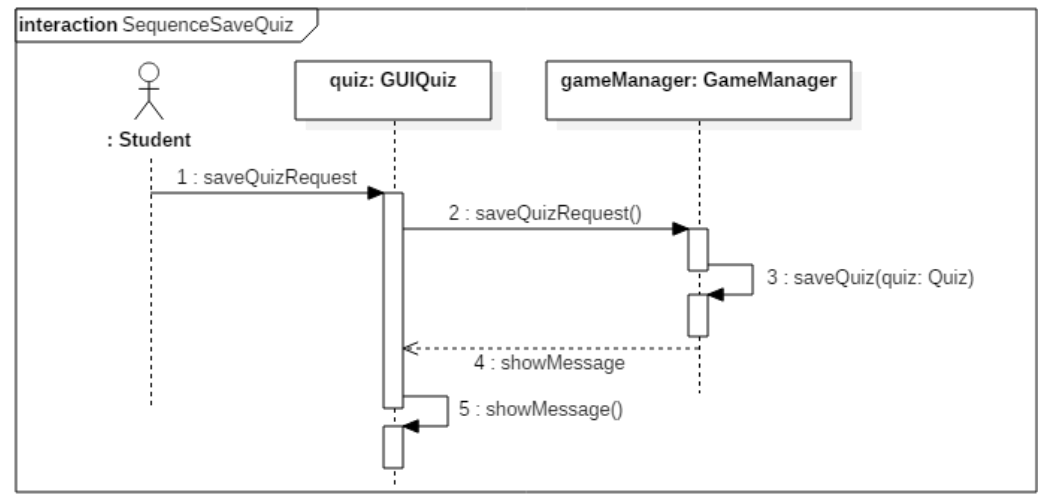

Fig. 5. Sequence diagram for sending knowledge recognition

\subsubsection{Presentation layer}

Finally, in the presentation layer, the Unity video game engine was used to visualize the skeletal system with each of its components, traumas and its descriptions, the display of visual effects and the interaction with the apprentice for each of the defined functionalities . For this purpose, the use of the software development kit (SDK) Vuforia for the generation of augmented reality effects was incorporated. Fig. 6 shows the class diagram of the presentation layer and its interaction with the business logic layer. 


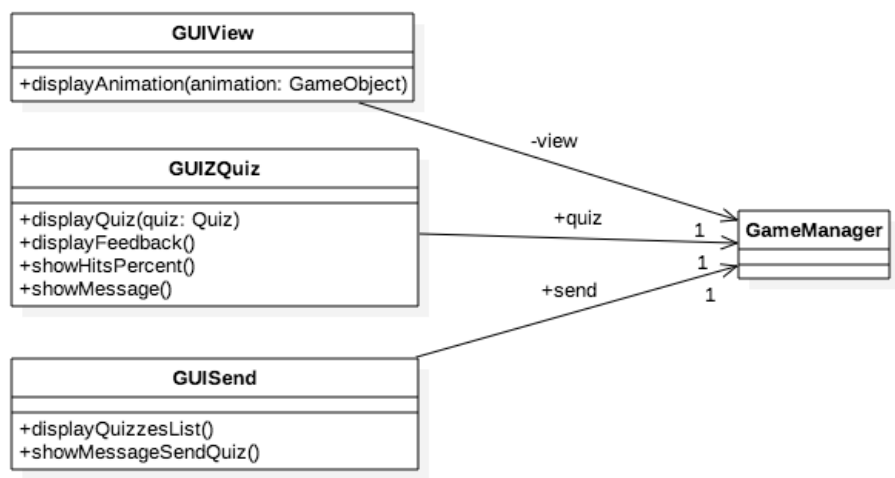

Fig. 6 Class diagram of the presentation layer

\subsection{Application}

The name of the application developed is AR4BI (Augmented Reality for Bone Injuries). The program begins by presenting a view configured for generic Cardboard lenses, ready to recognize the patterns that will lead, either to the visualization of bone injury models with their description, or to the evaluation of knowledge about them. The application, then, allows the recognition of the proper patterns of each reference point for various bones. At the time of entering the learning section, the user selects one of the bones of the body and the system teaches the different peculiarities of the bone lesions that can occur on it, by means of three-dimensional animations, narrations and interaction with the same.

On the other hand, at the time of entering the knowledge recognition module, the system randomly presents 1 lesion with 3 possible answers, the user must select one and when doing so, the system will present another lesion until completing 10 lesions. Once the Follow-up, the system shows the percentage of correct answers and the alternative of saving the result or sending it by email. Fig. 7 shows a screenshot of the teaching mode display (for Cardboard) where the user has to select a bone for the system to present the three-dimensional model together with the description of possible injuries on it. On the other hand, Fig. 8 illustrates the visualization mode of recognition of knowledge in which there is a fracture of the ulna and diaphyseal radius and the response options.

The application runs locally and performs data persistence in the same way in a relational database. It does not require connectivity to application or database servers during its execution. This can be executed on mobile devices with Android operating system since version 4.4 and iOS since version 9.0. Likewise, it allows its execution on the Web platform and local execution on desktop computers. 


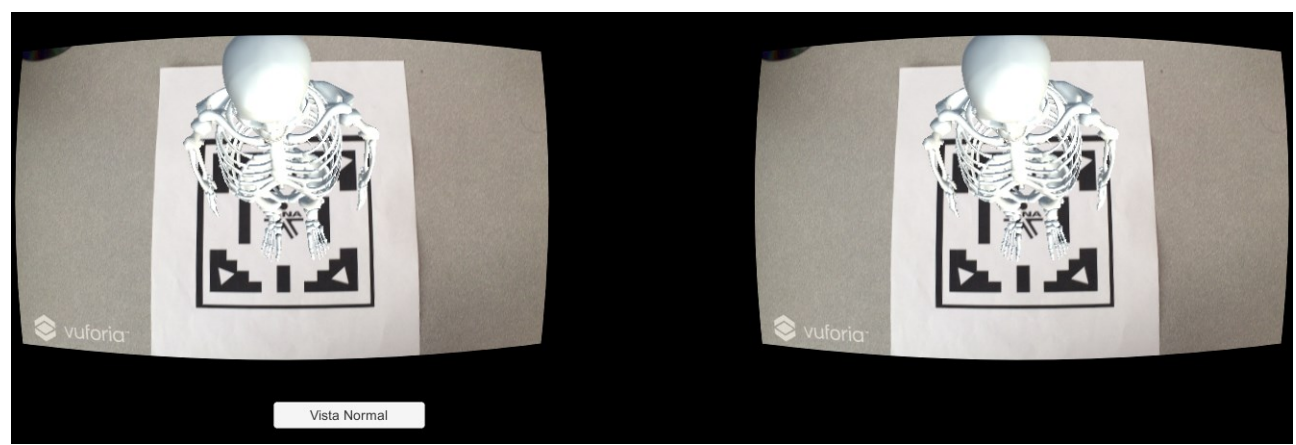

Fig. 7 Display screen of the teaching mode

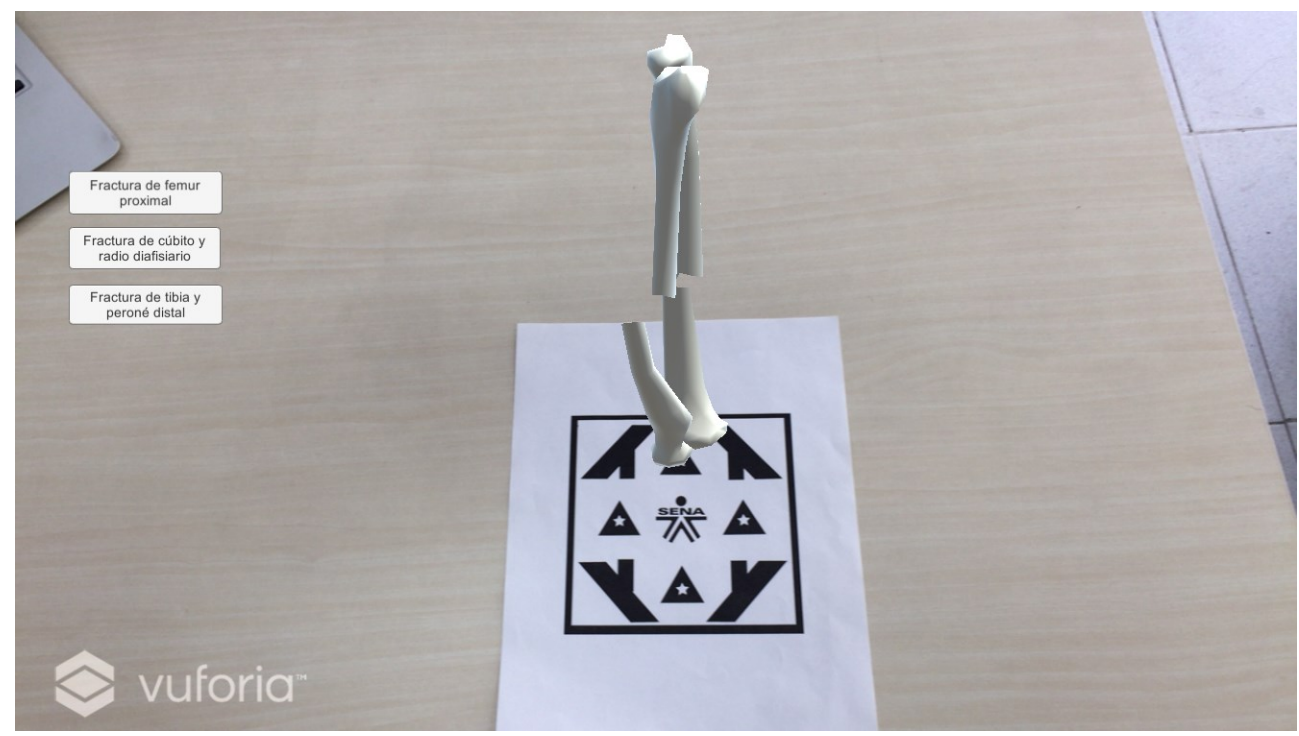

Fig. 8 Display screen of knowledge recognition mode

In the two previous figures we can see a watermark with the vuforia logo insofar as its free version was used.

\section{Conclusions and future work}

The Service and Trade Center of the National Training Service SENA in Tolima was given an easily accessible technological tool that allows improving its pedagogical processes in the face of cases of multiple injuries and fractures according to the concept of expert doctors in the area. 
The information system developed for the teaching and learning of bone injuries through augmented reality AR4BI, can positively impact the way in which new students in the health area are educated, because it strengthens interactive didactics using attractive technology for the new generations. However, this has to be the reason for future research, which undoubtedly has to be done before the software can be introduced definitively into a curriculum.

\section{Acknowledgements}

The authors would like to thank financial support from Trade and Services Center (Centro de Comercio y Servicios) of the National Training Service (Servicio Nacional de Aprendizaje SENA) from Colombia Project SGPS-2444-2017 from the System of Research, Technological Development and Innovation (SENNOVA).

\section{References}

Azuma, R. (1997). A survey of augmented reality. Presence-Teleoperators and Virtual Environments, 6(4), 355-385.

Bulstrode, C., Buckwalter, J., Carr, A., Marsh, L., Fairbank, J., Wilson-MacDonald, J., and Bowden, G. (2005). Oxford textbook of trauma and orthopaedics. New York: Oxford University Press.

Carretero González, J. (2010). Técnicas y recursos educativos en la enseñanza de la medicina. Educación médica, 13(Supl. 1), S9-S12. Retrieved 06 01, 2018, from http://scielo.isciii.es/scielo.php?script=sci_arttext\&pid=S1575-

$18132010000500003 \& \operatorname{lng}=$ es\&tlng=es

Furht, B., (2011). Handbook of Augmented Reality. New York: Springer.

Hopkins, L., Hampton, B. S., Abbott, J. F., Buery-Joyner, S. D., Craig, L. B., Dalrymple, J. L., ... Wolf, A. (2018). To the point: medical education, technology, and the millennial learner. American Journal of Obstetrics and Gynecology, 35(4), 954-963.

Kippe, G., and Rampolla, J. (2013). Augmented Reality: An Emerging Technologies Guide to $A R$. Waltham: Elseiver.

Klopfer, E., and Squire, K. (2008). Environmental detectives: the development of an augmented reality platform for environmental simulations. Educational Technology Research and Development, 56(2), 203-228. 
Kurup, V. (2010). The New Learners-Millennials!! International Anesthesiology Clinics, $48(3), 13-25$.

Twenge, J. M. (2009). Generational changes and their impact in the classroom: teaching Generation Me. Medical Education, 43, 398-405.

Wu, H.-K., Wen-Yu Lee, S., Chang, H.-Y., and Liang, J.-C. (2013). Current status, opportunities and challenges of augmented reality in education. Computers \& Education, 62(1), 41-49. 